\title{
Laparoscopic resection of hilar cholangiocarcinoma
}

\author{
Woohyung Lee, Ho-Seong Han, Yoo-Seok Yoon, Jai Young Cho, YoungRok Choi, Hong Kyung Shin, \\ Jae Yool Jang, Hanlim Choi
}

Department of Surgery, Seoul National University Bundang Hospital, Seoul National University College of Medicine, Seoul, Korea

\begin{abstract}
Laparoscopic resection of hilar cholangiocarcinoma is technically challenging because it involves complicated laparoscopic procedures that include laparoscopic hepatoduodenal lymphadenectomy, hemihepatectomy with caudate lobectomy, and hepaticojejunostomy. There are currently very few reports describing this type of surgery. Between August 2014 and December 2014, 5 patients underwent total laparoscopic or laparoscopic-assisted surgery for hilar cholangiocarcinoma. Two patients with type I or II hilar cholangiocarcinoma underwent radical hilar resection. Three patients with type IIla or IIlb cholangiocarcinoma underwent extended hemihepatectomy together with caudate lobectomy. The median (range) age, operation time, blood loss, and length of hospital stay were 63 years (43-76 years), 610 minutes (410-665 minutes), $650 \mathrm{~mL}$ (450-1,300 mL), and 12 days (9-21 days), respectively. Four patients had a negative margin, but 1 patient was diagnosed with high-grade dysplasia on the proximal resection margin. The median tumor size was $3.0 \mathrm{~cm}$. One patient experienced postoperative biliary leakage, which resolved spontaneously. Laparoscopic resection is a feasible surgical approach in selected patients with hilar cholangiocarcinoma.
\end{abstract}

[Ann Surg Treat Res 2015;89(4):228-232]

Key Words: Laparoscopy, Cholangiocarcinoma, Klatskin's tumor

\section{INTRODUCTION}

Hilar cholangiocarcinoma, also known as a Klatskin tumor, differs from other cholangiocarcinomas in term of the treatment method and prognosis. The 5-year survival rate was reported to range from $15 \%$ to $35 \%$, even after curative resection. Curative resection is often difficult because of invasion into adjacent important vessels and longitudinal extension along the bile duct in the submucosal layer. Curative surgical resection is the only treatment option that confers a long-term survival outcome in patients with hilar cholangiocarcinoma. The surgical procedure consists of extended hemihepatectomy, including caudate lobectomy, and regional lymphadenectomy. Because of the complexity of the surgical procedures, there have been very few reports on laparoscopic resection of hilar cholangiocarcinoma. However, accumulating experience and technical improvements in laparoscopic surgery have allowed surgeons to perform advanced laparoscopic procedures. The individual procedures of laparoscopic liver resection, laparoscopic hepatoduodenal lymphadenectomy, and laparoscopic hepaticojejunostomy, which are essential components of laparoscopic resection for hilar cholangiocarcinoma, have already been reported. However, there are currently very few reports describing laparoscopic resection of hilar cholangiocarcinoma, a combination of these three procedures. Therefore, the aim of this study was to evaluate the feasibility of performing laparoscopic resection of hilar cholangiocarcinoma.

\section{SURGICAL TECHNIQUE}

\section{Patients and indications for surgery}

Five patients who underwent laparoscopic resection of hilar
Received February 10, 2015, Revised May 26, 2015,

Accepted June 4, 2015

\section{Corresponding Author: Ho-Seong Han}

Department of Surgery, Seoul National University Bundang Hospital, 82 Gumi-ro 173beon-gil, Bundang-gu, Seongnam 13620, Korea

Tel: +82-31-787-7091, Fax: +82-31-787-4078

E-mail: hanhs@snubh.org
Copyright (C) 2015, the Korean Surgical Society

(c) Annals of Surgical Treatment and Research is an Open Access Journal. All articles are distributed under the terms of the Creative Commons Attribution NonCommercial License (http://creativecommons.org/licenses/by-nc/4.0/) which permits unrestricted non-commercial use, distribution, and reproduction in any medium, provided the original work is properly cited. 
cholangiocarcinoma between August 2014 and December 2014 were enrolled. The preoperative laboratory examinations included measurement of tumor markers. Preoperative biliary drainage was performed in 4 patients with jaundice, with surgery delayed until the total bilirubin level was $<2-3 \mathrm{mg} / \mathrm{dL}$. The types of Klatskin tumor and resectability were evaluated by preoperative computed tomography and magnetic resonance cholangiopancreatography. The present study was approved by Institutinoal Review Board of Seoul National University Bundang Hospital (No. B-1412-280-118).

\section{Operative procedures}

After general anesthesia, patients were placed in the lithotomy position and tilted into the $30^{\circ}$ reverse Trendelenburg position with right-side-up adjustment for right liver resection. The surgeon stood between the patient's legs. The scopist and assistant stood on the left side of the patient. A 12-mm camera port was placed in the subumbilical region. Pneumoperitoneum was established and maintained at $<13 \mathrm{mmHg}$. Two main working 12-mm ports were inserted in the subcostal area along the midclavicular line and anterior axillary line, respectively. Two additional ports were placed in the epigastric area and left upper quadrant. A flexible laparoscope was used in all procedures. After performing laparoscopic hepatoduodenal lymphadenectomy using ultrasonic shears (Harmonic scalpel, Ethicon, Cincinnati, OH, USA), the common bile duct was transected at the level of the superior border above the pancreas and the distal margin was evaluated using a frozen biopsy. In the case of Bismuth type I and II tumors, the proximal bile duct was transected over the confluence level and the margin was examined using frozen pathology. Bilioenteric anastomosis was performed using laparoscopic-assisted or total laparoscopic procedures. Laparoscopic-assisted anastomosis was performed through a 10-cm subcostal extension of a port site.

The resected specimen was retrieved through the wound after inserting the specimen into a protective bag. Ductoplasty was performed in patients with separated and numerous small hepatic ducts. After transecting the proximal jejunum $40 \mathrm{~cm}$ away from the Treitz ligament, Roux-en-Y hepaticojejunostomy was done in an antecolic fashion. End-to-side jejunojejunostomy was performed $50 \mathrm{~cm}$ away from the site of the hepaticojejunostomy. Total laparoscopic anastomosis was performed as follows. The proximal jejunum was transected using a laparoscopic stapler to create the Roux limb. After placing the Roux limb near the proximal resected hepatic duct, a small incision was made on the antimesenteric side of the jejunum. Interrupted or continuous laparoscopic sutures were placed from the posterior row to the anterior row using absorbable monofilament thread. Side-to-side jejunojejunostomy was performed along the antimesenteric side of the jejunum, and the common entry wound was closed using a laparoscopic

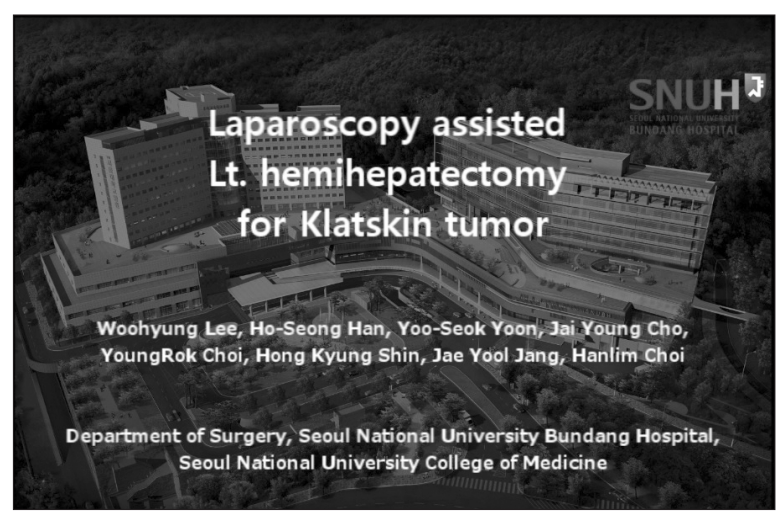

Fig. 1. Operative procedures (Video clip can be operated via http://www.astr.or.kr/src/sm/87-4_S001.wmv).

stapler. In the case of unilateral biliary involvement, such as Bismuth type IIIa or IIIb cholangiocarcinoma, we performed extended hemihepatectomy with caudate lobectomy. Laparoscopic Pringle's maneuver was performed in the case of sudden bleeding during liver parenchymal transection. After mobilization of the liver, the parenchyma was transected using a Cavitron Ultrasonic Surgical Aspirator (Integra Lifesciences, New Jersey, NJ, USA), including the caudate lobe. Bilioenteric anastomosis was performed as described above. After careful hemostasis, a fibrin glue sealant (Greenplast, Green Cross, Seoul, Korea) was applied to the cut surface of the liver. After irrigation of the surgical field, a silastic drain was placed around the anastomosis and the wound was closed layer by layer (Fig. 1).

\section{Patient characteristics and preoperative data}

Five male patients were enrolled. The median age of the patients was 63 years (range, 43-76 years). The preoperative American Society of Anesthesiologists score was 2 in 4 patients and 3 in 1 patient. All patients had preoperative jaundice, and 4 patients underwent preoperative biliary drainage. A percutaneous transhepatic biliary drain was inserted in 3 patients and endoscopic nasobiliary drainage was performed in 1 patient. Based on the preoperative imaging findings, Bismuth types I, II, IIIa, and IIIb cholangiocarcinoma were diagnosed in $1,1,1$, and 2 patients, respectively. The preoperative median (range) total bilirubin, CEA, and CA 19-9 concentrations were 3.7 $\mathrm{mg} / \mathrm{dL}(0.6-18.4 \mathrm{mg} / \mathrm{dL}), 3.1 \mathrm{ng} / \mathrm{mL}(2.2-10.3 \mathrm{ng} / \mathrm{mL})$, and $310 \mathrm{U} /$ $\mathrm{L}(26.2-1,000 \mathrm{U} / \mathrm{L})$, respectively (Table 1).

\section{Intraoperative, postoperative, and pathologic data}

Patients with Bismuth types I or II cholangiocarcinoma underwent laparoscopic hilar resection. The patient with Bismuth type I cholangiocarcinoma underwent total laparoscopic hilar resection with bilioenteric anastomosis, and the patient with Bismuth type II disease underwent total laparoscopic hilar resection and laparoscopic-assisted hepaticojejunostomy 
Table 1. Patient characteristics and preoperative data

\begin{tabular}{lc}
\hline \multicolumn{1}{c}{ Characteristic } & Value \\
Sex & \\
$\quad$ Male $:$ female & $5: 0$ \\
Age $(\mathrm{yr})$ & $63(43-76)$ \\
ASA score & \\
$1: 2: 3: 4: 5$ & $0: 4: 1: 0: 0$ \\
Jaundice & $5(100)$ \\
Preoperative biliary drainage & \\
$\quad$ PTBD $:$ ENBD $:$ ERBD & $3: 1: 0$ \\
Bismuth type & \\
I : II : Illa : IIIb : IV & $1: 1: 1: 2: 0$ \\
Preoperative total bilirubin $(\mathrm{mg} / \mathrm{dL})$ & $3.7(0.6-18.4)$ \\
Preoperative CEA (ng/mL) & $3.1(2.2-10.3)$ \\
Preoperative CA $19-9(\mathrm{U} / \mathrm{L})$ & $310(26.2-1,000)$ \\
\hline
\end{tabular}

Values are presented as number, median (range) or number (\%). ASA, American Society of Anesthesiologists; PTBD, percutaneous transhepatic biliary drainage; ENBD, endoscopic nasobiliary drainage; ERBD, endoscopic retrograde biliary drainage; CEA, carcinoembryonic antigen; CA 19-9, Carbohydrate antigen 19-9.

because the remaining bile ducts were separated into right anterior, posterior, and left bile ducts. Laparoscopic-extended hemihepatectomy with caudate lobectomy was performed in three patients, with the right lobe resected in one patient and the left lobe resected in two patients. The median (range) operation time, intraoperative blood loss, and postoperative hospital stay were 610 minutes (410-665 minutes), $650 \mathrm{~mL}$ (450-1,300 $\mathrm{mL}$ ), and 12 days (9-21 days), respectively. There were no deaths within postoperative 30 days. One patient experienced postoperative biliary leakage, which was managed using a percutaneous drain. The pathologic results showed that the median (range) tumor size was $3.0 \mathrm{~cm}(2.3-10.0 \mathrm{~cm})$. One patient had high-grade dysplasia at the proximal resection margin. All patients were followed up for a median of 8 months (range, 5-9 months). One patient experienced intrahepatic metastasis on 7 months after surgery (Table 2).

\section{DISCUSSION}

Because of recent developments in laparoscopic techniques and equipment, laparoscopic surgery has been positioned as an indispensable therapeutic modality in hepatobiliary and pancreatic surgery. However, laparoscopic resection of hilar cholangiocarcinoma is rarely performed, possibly because of the associated tumor characteristics, and requires complex laparoscopic procedures. Hilar cholangiocarcinoma arises from the biliary confluence, which is in close proximity to vascular structures. Invasion into the adjacent hepatic artery or portal vein make curative resection difficult. Longitudinal extension along the bile duct also affects the surgical outcomes [1]. Additionally, laparoscopic resection of hilar cholangiocarcinoma
Table 2. Intraoperative and postoperative data

\begin{tabular}{lc}
\multicolumn{1}{c}{ Variable } & Value \\
\hline Type of resection & \\
Hilar resection & 2 \\
Extended hemihepatectomy & 3 \\
$\quad$ Right : Left & $1: 2$ \\
Surgical time $(\mathrm{min})$ & $610(410-665)$ \\
Blood loss $(\mathrm{mL})$ & $650(450-1300)$ \\
Tumor size $(\mathrm{cm})$ & $3.0(2.3-10.0)$ \\
T stage & $1: 4: 0: 0$ \\
$1: 2: 3: 4$ & \\
N stage & $5: 0$ \\
$\quad 0: 1$ & $4(3-12)$ \\
Retrieved lymph nodes & 1 \\
Postoperative complications & $12(9-21)$ \\
Hospital stay (day) &
\end{tabular}

Values are presented as number, median (range) or number (\%).

requires several complex procedures, such as laparoscopicextended hemihepatectomy with caudate lobectomy, laparoscopic hepatoduodenal lymphadenectomy, and bilioenteric anastomosis, in order to achieve curative surgical resection.

Recent reports have described the feasibility and safety of these individual procedures performed in laparoscopic resection of hilar cholangiocarcinoma. Major laparoscopic liver resection is increasingly being performed in experienced centers. A fairly recent study showed that $26 \%$ of patients safely underwent major laparoscopic liver resection in Korea, and the procedure was associated with a similar open conversion rate and morbidity rate to a previous report [2]. Although caudate lobectomy is a challenging procedure, the number of reports describing surgical resection of these types is slowly increasing. Resection of the caudate lobe is essential for surgical resection of a Klatskin tumor. The caudate lobe ducts join the left and right hepatic ducts near their confluence. The anatomic relationship among these structures promotes tumor invasion around the caudate lobe. It was reported that patients who underwent surgery for hilar cholangiocarcinoma had microscopic tumor infiltration into the caudate lobe. Combining major liver resection and caudate lobectomy needs to be done by very experienced surgeons. Caudate lobectomy requires meticulous dissection, which can be accomplished using laparoscopic procedures. Several surgeons have also reported advantages of laparoscopic surgery for caudate lobe resection, which include improved visualization of a restricted area. Laparoscopic hilar lymphadenectomy and bilioenteric reconstruction are also necessary procedures. Lymph node dissection at the hepatoduodenal ligament is a very difficult procedure that also requires meticulous dissection. Laparoscopic dissection of the hepatoduodenal ligament is well documented in recent reports [3]. Several reports have 
Table 3. Summary of reports of laparoscopic resection for hilar cholangiocarcinoma

\begin{tabular}{|c|c|c|c|c|c|c|c|c|}
\hline Source & No. & $\begin{array}{c}\text { Hilar } \\
\text { resection }\end{array}$ & Hepatectomy & $\mathrm{CL}$ & $\mathrm{PP}$ & $\begin{array}{l}\text { Operation } \\
\text { time (min) }\end{array}$ & Blood loss (mL) & Complications (\%) \\
\hline Giulianotti et al. [7] & 1 & 0 & 1 & 0 & 0 & 549 & 800 & 0 \\
\hline Yu et al. [8] & 14 & 7 & 5 & 0 & 2 & 305 & 386 & 0 \\
\hline Machado et al. [5] & 1 & 0 & 1 & 0 & 0 & 300 & $\mathrm{NA}$ & 38 \\
\hline Gumbs et al. [10] & 5 & 0 & 5 & NA & 0 & NA & 240 & 0 \\
\hline Cho et al. [6] & 1 & 0 & 1 & 1 & 0 & 629 & 200 & 100 \\
\hline Present study & 5 & 2 & 3 & 3 & 0 & 580 & 725 & 20 \\
\hline
\end{tabular}

$\mathrm{CL}$, caudate lobectomy; $\mathrm{PP}$, palliative procedure; NA, not applicable.

also described the safety of laparoscopic hepatoduodenal lymphadenectomy and bilioenteric anastomosis for laparoscopic pancreaticoduodenectomy [4]. Furthermore, it is reported that there were no differences in oncologic outcomes or perioperative results compared with open procedures.

Despite the complexity of laparoscopic resection of hilar cholangiocarcinoma, our literature search revealed five prior reports describing this procedure. Machado et al. [5], Cho et al. [6], and Giulianotti et al. [7] each reported this procedure in one patient with hilar cholangiocarcinoma. Yu et al. [8] performed laparoscopic hilar resection in seven patients with Bismuth type I hilar cholangiocarcinoma, laparoscopic partial liver resection with hepaticojejunostomy in five patients with Bismuth type II disease, and palliative resection for advanced disease. However, they were criticized for performing partial hepatectomy without caudate lobectomy because of the technical difficulty and high recurrence rate after surgery [9]. Gumbs et al. [10] performed total laparoscopic resection of hilar cholangiocarcinoma in 5 patients; 3 patients underwent laparoscopic hilar resection and 2 patients underwent laparoscopic hemihepatectomy. In the present study, 5 patients underwent total laparoscopic and laparoscopic-assisted resection of hilar cholangiocarcinoma, and the perioperative results were comparable with those of the previous reports (Table 3).

There are still some unresolved problems regarding adequate laparoscopic resection of hilar cholangiocarcinoma. It is often difficult to select suitable cases for laparoscopic procedures.
Achieving an adequate intraoperative safety margin is another problem. Yu et al. [8] reported that 2 of 14 patients had a positive margin stated in their pathologic reports and Gumbs et al. [10] reported a case requiring $\mathrm{R} 1$ resection. In the present study, the pathologic report for one patient listed high-grade dysplasia. Nevertheless, despite the perceived difficulty associated with laparoscopic procedures, several studies have demonstrated the safety of each component of laparoscopic resection of hilar cholangiocarcinoma, and further experience of performing this procedure will provide further support for its use.

\section{CONCLUSION}

We believe that the present study demonstrates the feasibility of performing laparoscopic resection of hilar cholangiocarcinoma in selected patients.

\section{CONFLICTS OF INTEREST}

No potential conflict of interest relevant to this article was reported.

\section{SUPPLEMENTARY MATERIALS}

Supplementary material (video clip) can be found via http:// www.astr.or.kr/src/sm/87-4_S001.wmv.

\section{REFERENCES}

1. Han IW, Jang JY, Kang MJ, Kwon W, Park JW, Chang YR, et al. Role of resection for Bismuth type IV hilar cholangiocarcinoma and analysis of determining factors for curative resection. Ann Surg Treat Res 2014;87:87-93.
2. Hwang DW, Han HS, Yoon YS, Cho JY, Kwon Y, Kim JH, et al. Laparoscopic major liver resection in Korea: a multicenter study. J Hepatobiliary Pancreat Sci 2013; 20:125-30.

3. Han HS, Cho JY, Yoon YS, Hwang DW,
Kim YK, Shin HK, et al. Total laparoscopic living donor right hepatectomy. Surg Endosc 2015:29:184.

4. Kendrick ML, Cusati D. Total laparoscopic pancreaticoduodenectomy: feasibility and outcome in an early experience. Arch Surg 
2010;145:19-23.

5. Machado MA, Makdissi FF, Surjan RC, Mochizuki M. Laparoscopic resection of hilar cholangiocarcinoma. J Laparoendosc Adv Surg Tech A 2012;22:954-6.

6. Cho A, Yamamoto H, Kainuma O, Muto Y, Yanagibashi H, Tonooka T, et al. Laparoscopy in the management of hilar cholangiocarcinoma. World J Gastroenterol 2014:20:15153-7.
7. Giulianotti PC, Sbrana F, Bianco FM, Addeo P. Robot-assisted laparoscopic extended right hepatectomy with biliary reconstruction. J Laparoendosc Adv Surg Tech A 2010;20:159-63.

8. Yu H, Wu SD, Chen DX, Zhu G. Laparoscopic resection of Bismuth type I and II hilar cholangiocarcinoma: an audit of 14 cases from two institutions. Dig Surg
2011;28:44-9.

9. Donati M, Stavrou GA, Oldhafer KJ. Laparoscopic resections for hilar cholangiocarcinomas: a critical appraisal. Dig Surg 2011;28:277-8.

10. Gumbs AA, Jarufe N, Gayet B. Minimally invasive approaches to extrapancr eatic cholangiocarcinoma. Surg Endosc 2013; 27:406-14. 